\title{
Fabrication of biosensors by attachment of biological macromolecules to electropolymerized conducting films
}

\author{
S. Cosnier and Ch. Gondran
}

Laboratoire d'Électrochimie Organique et de Photochimie Rédox, UMR CNRS 5630, Université Joseph Fourier Grenoble 1, BP 53, 38041 Grenoble Cedex 9, France

The concept and potentialities of electrochemical procedures of biomolecule immobilization are described. This method of biosensor construction is based on the electrogeneration of polymer films functionalized by specific groups allowing subsequently the attachment of biomolecules. The biomolecule immobilization on these films by covalent binding or affinity interactions are described.

\section{Introduction}

The ingenious concept to combine the recognition properties of macromolecular biological molecules to the sensitivity of electrochemical devices, has lead to the emergence of biosensors as valuable analytical tools for the monitoring of target analytes in different technological areas. For three decades biosensors have been the subject of increasing research effort and now constitute a major component of mainstream analytical chemistry $[1,2]$.

In recent years, there has been a growing interest in the development of microfabricated electrochemical biosensors. However, the stable and reproducible immobilization of macromolecular biomolecules such as enzyme, oligonucleotide or antibody, on conductive microsurfaces with complete retention of their recognition properties remains a crucial problem for the commercial development of miniaturized biosensors. Effectively, most of the conventional procedures of biomolecule immobilization such as cross-linking, covalent binding and entrapment in gels or membranes suffer from a low reproducibility and a poor spatially controlled deposition.

A part from these conventional methods, the immobilization of biomolecules in or on electropolymerized films is gaining importance [3-5]. One major advantage of electrochemical deposition procedures is the possibility of precisely electrogenerating a polymer coating over conductive microsurfaces of complex geometry. In addition the polymer films exhibit a good robutness in operation in aqueous and organic media. The electrochemical entrapment of biomolecules in organic polymers involves the application of an appropriate potential to the working electrode soaked in aqueous solution containing both biomolecule and electropolymerizable monomer (Fig. 1). Biomolecules present in the immediate vicinity of the electrode surface are thus incorporated in the growing polymer. In addition, this entrapment occurs without chemical reaction that could affect the activity of the entrapped biomolecules. The advantage of the electrochemical polymerization is that films can be prepared easily in a rapid one-step procedure. Furthermore, this method enables exact control of the thickness of the polymer layer based on the measurement of the electrical charge passed during the electrochemical polymerization. Most of the electrochemically deposited polymers used for the biomolecule immobilization are conducting polymers since their formation (contrary to a non-conducting polymer) is not restricted to the elaboration of very thin films. Among these conducting

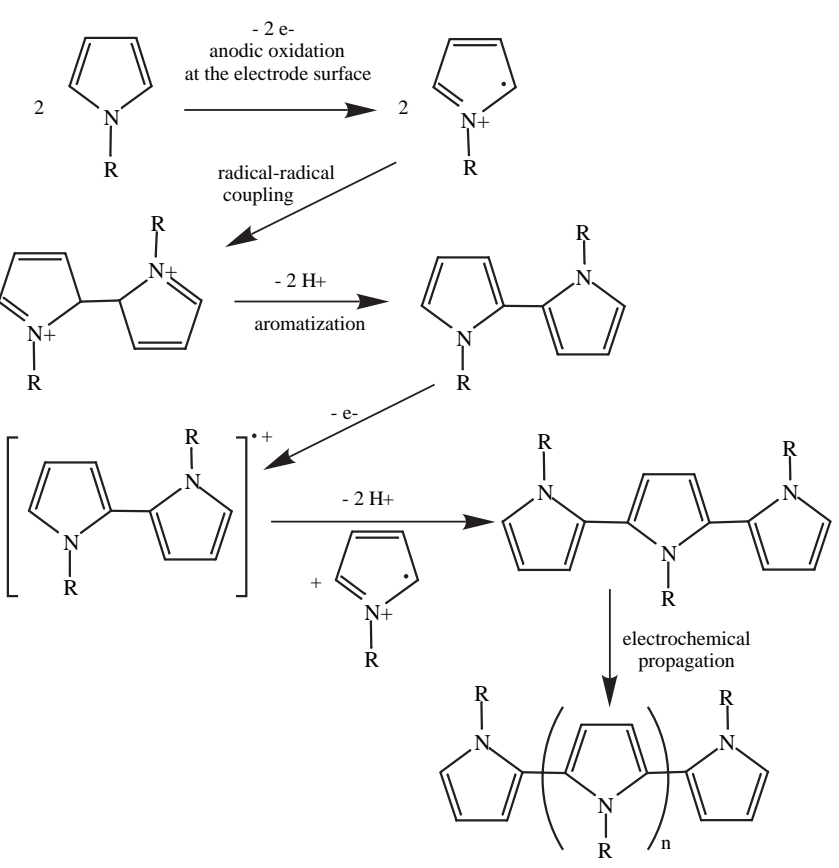

Figure 1. Electrochemical generation of functionalized polypyrrole films (polypyrrole : $R=H$ ). 


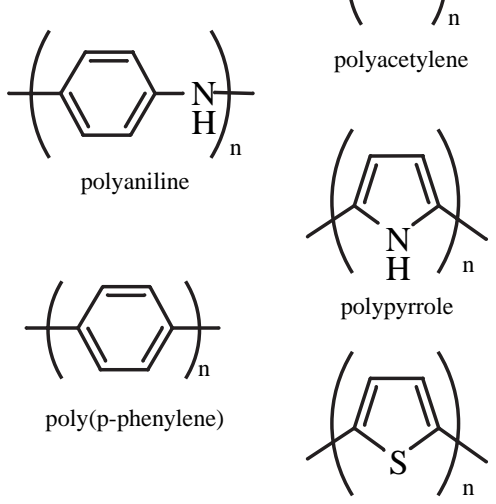

polythiophene

Figure 2. Conducting polymer films used for the entrapment of biological macromolecules.

polymers like polyacetylene, polythiophene and polyaniline, polypyrrole and its derivatives play the leading role due to their versatile applicability (Fig. 2).

Among the different biomolecules, the entrapment within polypyrrole matrices has been mainly focused on enzyme molecules, particularly oxidases. Nevertheless, few examples have been devoted to the immobilization of coenzymes, antibodies, cells and biological tissue, illustrating the wide potentialities of this method for biomolecule retention [6-8]. The most widely-held opinion is that the immobilization of biomolecules in electrogenerated polymers is partly due to electrostatic interactions between negatively or positively charged proteins and polymer films, the proteins being incorporated within the polymer as counter-ions. However, most often the electropolymerization process requires very high concentrations of a supporting electrolyte or a buffer. As a result the incorporation of protein molecules in the growing polymer film is more likely due to the presence of protein molecules in the immediate vicinity of the electrode surface and does not result from specific electrostatic interactions. Moreover, the biomolecule immobilization by electrochemical entrapment in conducting or non conducting polymer films requires also high concentrations of biomolecule during the electropolymerization process.

An original strategy to overcome this drawback involved the immobilization of monomer and enzyme together, by adsorption, on the electrode surface before the electropolymerization step. Taking advantage of the adsorption and electropolymerization properties of amphiphilic pyrrole monomers, an original two-step procedure of biosensor construction has been developed [9-11]. This strategy of enzyme immobilization is based first on the solubilization of biomolecule in an aqueous dispersion of amphiphilic pyrrole. Then the aqueous mixture is spread and dried on an electrode surface. The electropolymerization of the adsorbed monomers in an aqueous electrolyte provides the irreversible entrapment of biomolecule in the resulting polypyrrolic matrix.

However, the physical entrapment of proteins within electrogenerated polymers induces a marked loss of their biological activity. This loss could be attributed to the hydrophobic character of the organic polymers [12]. Moreover, the steric constraints generated by the surrounding polymer drastically reduce the accessibility to the immobilized biomolecules. In addition, these constraints may hinder the formation of specific antigen-antibody binding or the hybridization of complementary oligonucleotides. Consequently, the electrochemical entrapment of proteins in polymeric films seems to be an inappropriate approach for the fabrication of immunosensors.

Another electrochemical method involves, initially, the electropolymerization of functionalized conducting polymers. Then the attachment of biomolecules to the polymer surfaces can be obtained by chemical grafting or by affinity of the biomolecule at the functional group. In comparison to the physical entrapment of biomolecules within polymer films such as polypyrrole, polythiophene, polyacetylene or polyaniline, this approach preserves a better access of substrate to the immobilized biomolecules and facilitates macromolecular interactions.

In this article, the principle and advantages of this electrochemical method of biomolecule attachment for the construction of biosensors will be reviewed.

\section{Attachment of biomolecules to electrogenerated organic polymers}

\section{Covalent binding of biomolecules at conducting polymer films}

An attractive alternative to the biomolecule entrapment involves the covalent binding of the biomolecule to polymer films bearing adequate functional groups. The main advantage of this sequential procedure, namely electropolymerization and covalent binding, lies in the possibility to use optimal conditions for each step. In particular, the initial formation of polymer films can be performed under conditions (organic solvents, high potential values for the polymerization process, reactivity of the generated radical monomers) which are deleterious for biomolecules. Moreover, the covalent binding of the biomolecule to the functionalized polymer can be carried out in aqueous buffer solutions containing additives and stabilizers which preserve the catalytic activity and/or the recognition properties of the biomolecule.

Schuhmann et al. first reported an original four-step procedure of enzyme immobilization, namely polymer electrogeneration, chemical and electrochemical post-functionalization of the polymer and then chemical grafting of the enzyme [13-15]. For example, the chemical nitration of an electropolymerized polypyrrole film was followed by the 
<smiles>Nc1cc2cccccc-2c1</smiles><smiles>Nc1cc(-c2cccs2)ccc1-c1cccs1</smiles><smiles>Nc1ccc(-n2c(-c3cccs3)ccc2-c2cccs2)cc1</smiles><smiles>NCCn1c(-c2cccs2)ccc1-c1cccs1</smiles>

Figure 3. Structures of amino-substituted monomers.

electrochemical reduction of the in situ generated nitro groups into amino groups. After activation of the carboxylic groups of glucose oxidase by carbodiimine, the enzyme molecules were covalently bound to the polypyrrole film through amide bonds. This approach of enzyme immobilization presents the main advantage to generate electrochemically the amino groups necessary for the enzyme grafting. Therefore, several biomolecules can be specifically immobilized onto different selected microelectrodes among microelectrodes arrayed on a silicon chip [4]. More recently, a potentiometric biosensor for urea was thus prepared by covalent attachment of urease to a polypyrrole film bearing electrogenerated amino groups [16]. However, these successive functionalizations may lead to inhomogeneously substituted polypyrrole films. In order to circumvent this drawback, the electropolymerization of pyrroles, azulenes, dithienylpyrroles and dithienylbenzene derivatives functionalized by an amino group has been exploited (Fig. 3) [17, 18]. The subsequent coupling of biomolecules to the polymerized amino groups was performed by activation of carboxylic groups of the protein shell of the biomolecule via a water-soluble carbodiimine. A similar approach has been developed with the electropolymerization of thiophene-3-acetic acid. The enzyme, nitrate reductase (NR), has been immobilized by covalent linkage between its lysine residues and the pendent carboxylic groups of the polythiophene matrix [19]. In addition, the copolymerization of thiophene-3-acetic acid and thiophene-bipyridinium allows simultaneously the covalent binding of NR and its electrical wiring [19].

In order to prepare mediated amperometric biosensor, the electrogeneration of polypyrrole films bearing carboxylic groups has been carried out by a pulse technique [20]. Phenothiazine dyes have thus been covalently bound to the polymerized carboxylic groups to mediate the reduction of quinoid products. The latter were enzymically produced by a polyphenol oxidase entrapped within an outer polypyrrolic layer [21]. In the same vein, an electropolymerized polypyrrole film functionalized by carboxylic groups was prepared to establish a direct electronic communication with cytochrome C, a redox protein [22]. More recently, Saby and Luong reported an unusual enzyme immobilization procedure based on an adhesive protein isolated from Mytilus edulis blue mussels [23]. The adhesive protein exhibits a high L-Dopa content (10-15\%). The oxidation of these catecholic amino acids provides highly reactive groups towards the amino groups of the protein backbone. Consequently, the electrooxidation of the adsorbed protein molecules induces a cross-linking process and hence the formation of a protein film on various electrode surfaces such as platinum, gold and glassy carbon. In addition, the covalent attachment of enzyme via its amino groups to the surface of the protein film can be performed following the same process [23].

However, the post-functionalization of polymer films bearing amino or carboxylic groups requires reaction conditions which often could partly denature the protein and/or lead to incomplete derivatization of the polymer surface. Consequently, the development of more effective and selective procedures for the biomolecule coupling to functionalized polymers has been recently investigated. Since activated esters can form, under very mild conditions, amide bond with derivatives containing amino groups, a series of electropolymerizable monomers functionalized by activated esters have been prepared [24-26]. Thus, the electrochemical polymerization of thiophene, bithiophene and pyrrole derivatives substituted by easy leaving groups (N-hydroxysuccinimide and N-hydroxyphtalimide esters has led to attractive precursor polymers (Fig. 4). It should be noted that the pyrrolic monomers were substituted on their bêta position in order to improve the conducting properties of the resulting polypyrrole. The latter could exhibit a more regular polymeric structure than that of N-substituted polypyrrole films [27]. Functionalized polythiophene and poly(bithiophene) films have been applied to the immobilization of glucose oxidase (GOx) through the reaction of the polymerized activated ester groups with the amino

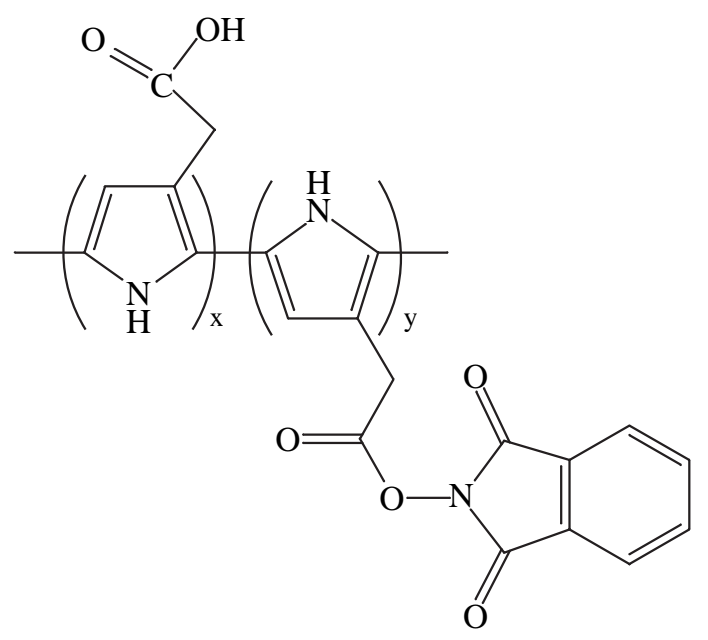

Figure 4. Structure of a precursor polymer film : poly [(3-acetic acid pyrrole)/(3-N-hydroxyphthalimide pyrrole)]. 
groups of the protein shell. The resulting biosensors present a current response for glucose which increases with increasing film thickness and then decreases for thick films [24]. This effect may be attributed to an initial increase in the roughness of the polymer film. On the other hand, the precursor polypyrrole film has been successfully functionalized by an oligonucleotide. The specific hybridization of this grafted oligonucleotide with its complementary nucleotide target induces a drastic modification in the electrochemical behaviour of the conductive polypyrrole backbone. This phenomenon has been exploited to build a very sensitive electrochemical sensor for oligonucleotides, the detection limit being $10^{-11} \mathrm{~mol}$ of oligonucleotide in solution. It should be noted that the electrochemical response of the polypyrroleoligonucleotide electrode remains unchanged after incubation in buffered aqueous solutions containing non complementary oligonucleotides. Owing to its specificity and simple use, it is expected that this kind of electrochemical biosensor will be useful for the detection of DNA sequences.

\section{Attachment of biomolecules by affinity with functionalized polymer films}

The chemical step in the procedure of biomolecule immobilization does not allow to preselect convenient positions on the protein shell for the covalent binding and hence to preserve the active site. Consequently the chemical step was replaced by an approach based on affinity interactions which may induce a regioselective grafting.

The functionalization of pyrrole monomers with bioactive dipeptides has led to electroactive poly (dipeptide-pyrrole) films which exhibit specific recognition properties towards proteolytic enzymes [28]. Thus, the possible enzyme binding to the functionalized polypyrrole film was examined with carboxypeptidase A. This enzyme forms a stable complex with the polymerized dipeptide residues which act as an enzyme inhibitor. In the same vein, the electropolymerization of antigenic peptides functionalized by 3-hydroxyphenylacetic acid as polymerizable groups has been realized for the specific recognition of antibodies [29]. The successful binding of the antibodies to the polymer film illustrates the accessibility of the polymerized peptides. However, the maximum amount of biomolecules coupled to the polymer films is always restricted to the formation of a complete molecular monolayer at the interface polymersolution.

A more promising strategy of biomolecules attachment which preserves their biological activity consist of using the simple formation of avidin-biotin bridge with biotinylated biomolecules or avidin-conjugated biomolecules. The protein avidin will bind 4 biotins, a vitamin, by a non-covalent interaction which is quasi-irreversible. Because of the high affinity of the avidin-biotin interaction (association constant $\mathrm{K}_{\mathrm{a}}=10^{15} \mathrm{M}^{-1}$ ) [30], this coupling system has been extensively used for the biomolecule immobilization [31, 32]. In addition, the formation of avidin-biotin bridges allows the construction of biomolecule multilayers. In this context, an innovative electrochemical method of biomolecule immobi-

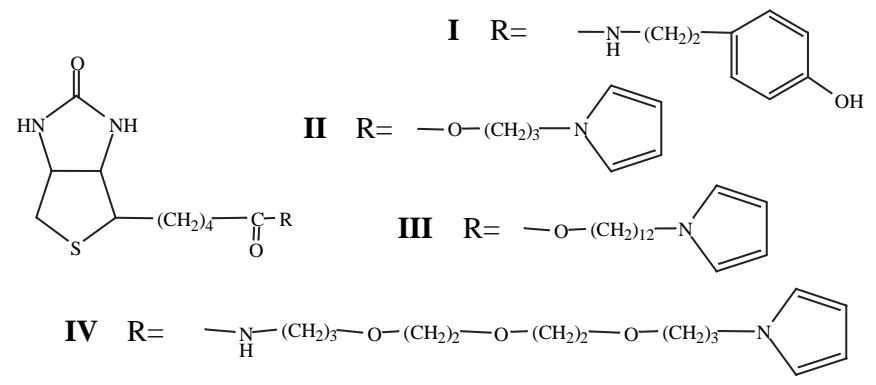

Figure 5. Structures of biotin derivatives substituted by an electropolymerizable phenol or pyrrole group.

lization consists in the electrogeneration of biotinylated polymer films. Recently, the formation of biotinylated surfaces has been attempted by electropolymerization of a biotin derivative functionalized by a phenol group (Fig. 5) [33]. The efficient coupling of an avidin-conjugated $\beta$-galactosidase to the biotinylated film has shown that this kind of polymer films provides biotin sites able to develop specific interactions with avidin. Jung and coworkers have also prepared electropolymerized polyphenol films containing biotin residues [34]. The availability of the polymerized biotin was confirmed with fluorescein-labeled streptavidin. Unfortunately, the electropolymerization provides nonconductive films which passivate the electrode surface and prevent further film growth. In addition their formation is restricted to very thin films. Consequently, the protein coupling could decrease the polymer stability.

An alternative consists in the electrogeneration of electronic conducting polymers functionalized by biotin moieties. In particular, their conductivity allows the reproducible formation of polymer layers of electrochemically controlled thickness. With this aim in view, biotin derivatives bearing an electropolymerizable pyrrole group have been recently synthesized and applied to the elaboration of electrically conducting polypyrrole films [35-38] (Fig. 5). However, owing to the poor electropolymerization ability of these biotin derivatives, the polymer formation requires a copolymerization process with free pyrrole [36], a pyrrole ammonium [37] or a polypyridinyl complex of ruthenium containing three pyrrole groups [35]. Nevertheless, a pure biotinylated conducting polypyrrole film can be electrogenerated from the biotin derivative III. The improved electropolymerization ability of this pyrrole biotin may be related to the increase in the length of the alkyl spacer between pyrrole and biotin residues. Moreover, the successful copolymerization of the biotin derivative II with a polypyridinyl complex of ruthenium functionalized by pyrrole groups provides redox copolymers. The stable redox conductivity at high anodic potentials due to the electropolymerized ruthenium complex $\left(\mathrm{Ru}^{\mathrm{II} / I I I}\right.$ couple) enables the sustained film growth [35].

The efficient fixation of avidin onto a biotinylated polypyrrole film was investigated by quartz microbalance 


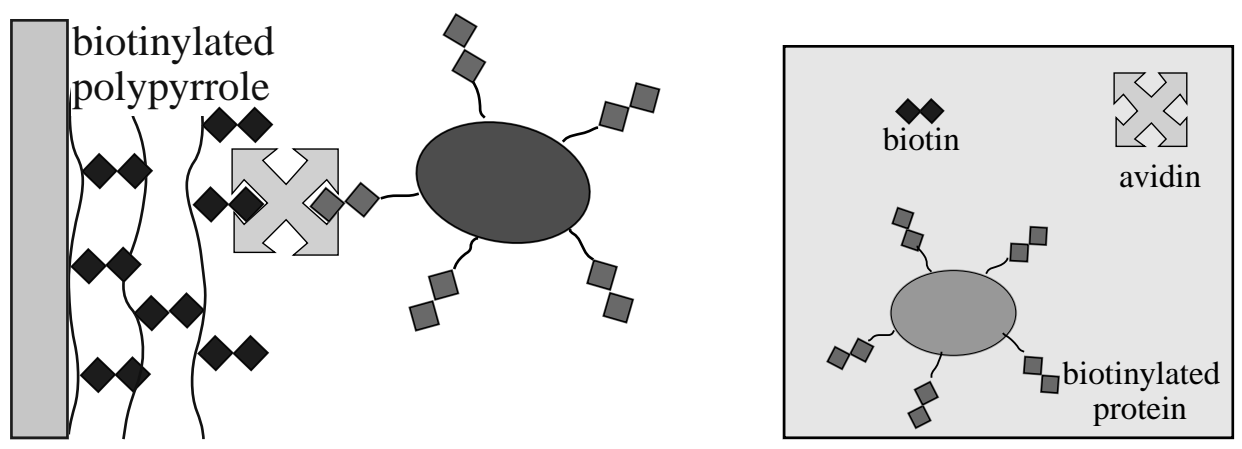

Figure 6. Schematic configuration of a biotinylated polypyrrole film functionalized by a protein monolayer via avidin-biotin bridges.

experiments [38]. In the presence of an aqueous solution of avidin, a rapid variation in frequency is clearly recorded for a quartz modified by a biotinylated polypyrrole film. This corresponds to an increase in mass of $550 \mathrm{ngcm}^{-2}$. Taking account that the theoretical maximum coverage of an avidin monolayer was estimated as $5.48 \times 10^{-12} \mathrm{~mol} \mathrm{~cm}^{-2}$ [39], the corresponding increase in mass is $362 \mathrm{ng} \mathrm{cm}^{-2}$. Therefore, the biotinylated polypyrrole film is able to bind efficiently 1.5 avidin monolayer [38].

Owing to the remarkable high affinity of avidin for biotin coupled to proteins or nucleic acids the attachment of biotinylated enzymes on biotinylated polypyrrole films has been investigated (Fig. 6). For this purpose, a biotinylated glucose oxidase (GOx-B) was chosen as enzyme model. Thus the soft anchoring of biotinylated macromolecular biomolecules via avidin-biotin bridge has been examined by permeation measurements. For this purpose, the evolution of the polymer permeability for the successive binding of avidin and GOx-B has been investigated by recording the cyclic voltammogram of ferrocene carboxylic acid (Fc) used as a permeant electroactive probe (Fig. 7). Since the electrochemical responses of the probe and polypyrrole backbone are close together, the polypyrrole electroactivity was destroyed by overoxidation to avoid mixing of the two signals. The cyclic voltammograms for Fc at an electrode modified by a biotinylated polypyrrole clearly show that the current peaks for the oxidation of $\mathrm{Fc}$ decreases after the immersion of the modified electrode into an avidin solution. In addition this signal is markedly reduced after the subsequent immersion into a GOx-B solution (Fig. 7). This decrease in the oxidation on the underlying glassy carbon electrode of Fc which diffused through the film, clearly indicates a permeability diminution attributed to an increase in the polymer thickness. This illustrates successively the binding of avidin to the polymerized biotin moities and that of GOx-B to avidin.

It has been previously reported that avidin-biotin interactions can be used successfully to build controlled molecular architectures composed of multienzyme layers [32, 40, 41]. Therefore the biotinylated polypyrrole electrodes have been applied to the construction of multienzyme layers by suc- cessive immersion of the modified electrodes into an avidin solution and then into GOx-B solution. Following this easy and fast procedure, several enzyme layers were fixed on the polymer surface. Since GOx-B catalyzes the oxidation of glucose with the production of hydrogen peroxide, the determination of glucose was carried out through the electrochemical oxidation of $\mathrm{H}_{2} \mathrm{O}_{2}$ at the electrode surface. Figure 8 shows the resulting calibration curves for glucose obtained with biosensors based on one, two and three enzyme layers. It appears clearly that the biosensor sensitivity for glucose increases approximatively with the number of enzyme layer namely $0.79,1.65$ and $2.16 \mathrm{~mA} \mathrm{M}^{-1} \mathrm{~cm}^{-2}$ for monolayer, 2layers and 3-layers respectively.

This demonstrates the successive elaboration of reproducible enzyme layers [35]. The synthesis of a biotin linked

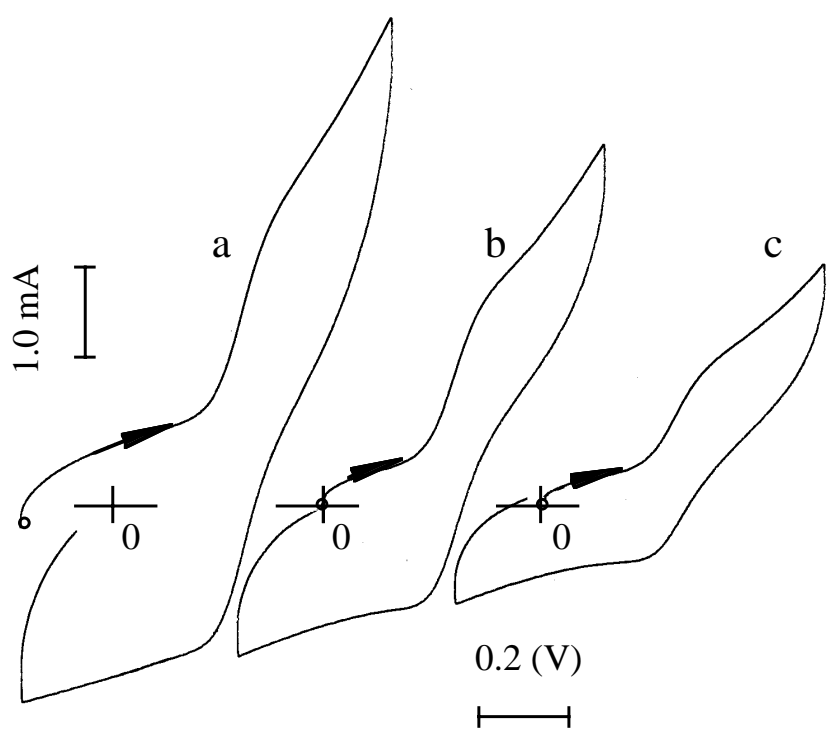

Figure 7. Cyclic voltammograms of $1 \mathrm{mM}$ ferrocene carboxylic acid in $\mathrm{H}_{2} \mathrm{O}+0.1 \mathrm{M} \mathrm{LiClO}_{4}$ at a glassy carbon electrode, diameter $3 \mathrm{~mm}$, modified by (a) a biotinylated polypyrrrole film (b) after immersion in an avidin solution and (c) after immersion in a biotinylated glucose oxidase solution; scan rate $0.1 \mathrm{Vs}^{-1}$. 

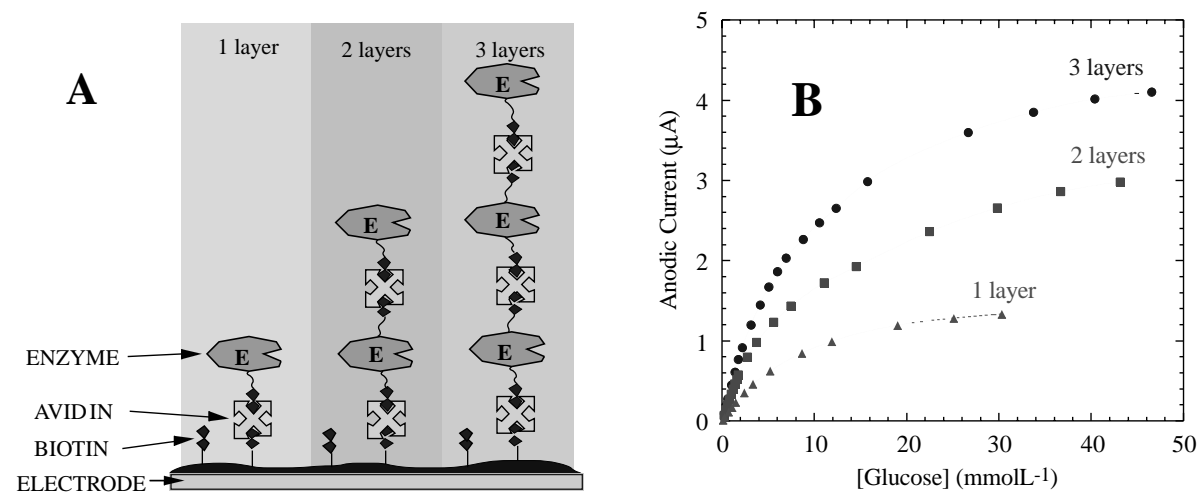

Figure 8. A Scheme of the biosensor configuration. B Calibration curves for glucose obtained with biotinylated copolymer electrodes modified by a monolayer, two layers and three layers of GOx-B. Applied potential $0.6 \mathrm{~V}$ vs SCE; air saturated 0.1M phosphate buffer ( $\mathrm{pH} \mathrm{7)}$ kept under stirring.

by a long hydrophilic arm to an electropolymerizable pyrrole group (IV) has been recently reported (Fig. 5) [36]. The improvement in monomer solubility in water allows the electropolymerization of this biotin derivative in aqueous solutions. In addition, the hydrophilic arm may facilitate the accessibility to the polymerized biotin groups. The efficient immobilization of streptavidin-conjugated $\mathrm{R}$ phycoerythrin on biotinylated films obtained by copolymerization of pyrrole and IV, was demonstrated by fluorescence measurements. It should be noted that the intensity of the fluorescence can be related to the amount of biotin derivative involved in the copolymerization process.

\section{Conclusion}

Among the numerous procedures of protein immobilization, the attachment of macromolecular biomolecules to electropolymerized films constitutes a simple method for the functionalization of conductive microsurfaces. In addition the use of functionalized polymers bearing easy leaving groups or recognition sites for the chemical grafting or the selective complexation of biomolecules, has opened interesting perspectives in the field of biosensor construction. In particular, owing to the commercial availability of a large variety of biotinylated biomolecules and avidin-conjugated biomolecules, the electrogeneration of biotinylated polymers seems to be a promising avenue for the development of microbiosensors and immunosensors. Furthermore, this approach should allow the construction of controlled architectures based on spatially segregated multilayers, exhibiting complementary biological activities.

\section{Acknowledgement}

We thank Dr. A. Deronzier for continuous support in the field of electrochemical biosensors.

\section{References}

1. Turner, A.P.F.; Karube, I.; Wilson, G.S. Biosensors : fundamentals and applications; Oxford University Press: New York, 1987.

2. Kauffmann, J.M.; Guilbault, G.G. In : Bioanalytical applications of enzymes, John Wiley \& Sons, Inc, 1992, Vol 36.

3. Bartlett, P.N.; Cooper, J.M. J. Electroanal. Chem. 1993, 363, 1-12.

4. Schuhmann, W. Mikrochim. Acta 1995,121, 1-29.

5. Trojanowicz, M.; Krawezynski vel Krawcyk, T. Mikrochim. Acta 1995, 121, 167-181.

6. Deshpande, M.V.; Hall, E. A. H. Biosens. Bioelectron. 1990, 5, 431-448.

7. John, R.; Spencer, M.; Wallace, G.G.; Smyth, M.R. Anal. Chim. Acta 1991, 24, 381-385.

8. Uchiyama, S.; Hasebe, Y.; Tanaka, M. Electroanalysis 1997, 9, 176-178.

9. Cosnier, S.; Innocent, C. J. Electroanal. Chem. 1992, 328, 361-366.

10. Cosnier, S. Electroanalysis 1997, 9, 894-902.

11. Cosnier, S. Can. J. Chem. Eng, 1998, 76,1000.

12. Cosnier, S.; Le Pellec; Guidetti, B.; Rico-Lattes, I. J. Electroanal. Chem. 1998, 449,165-171.

13. Schuhmann, W.; Lammert, R.; Uhe, B.; Schmidt, H.-L. Sensors and Actuators B 1990, 1, 537-541.

14. Schuhmann, W. Synth. Met. 1991, 41-43, 429-432.

15. Schuhmann, W. Sensors and Actuators B 1991, 4, 41-49.

16. Hernandez, E. C.; Witkowski, A.; Daunert, S.; Bachas, L.G. Mikrochim. Acta 1995, 121, 63-72.

17. Schuhmann, W.; Huber, J.; Mirlach, A.; Daub, J. Adv. Mater. 1993, 5, 124-126.

18. Röckel, H.; Huber, J.; Gleiter, R.; Schuhmann, W. Adv. Mater. 1994, 6, 567-571.

19. Willner, I.; Katz, E.; Lapidot, N.; Baüerle, P. Bioelectrochem. Bioenerg. 1992, 29, 29-45.

20. Schuhmann, W.; Krang, C.; Wohlschläger, H.; Strohmeier, J. Biosens. Bioelectron. 1997, 12, 1157-1167. 
21. Kranz, C.; Wohlschläger, H.; Schmidt, H.L.; Schuhmann, W. Electroanalysis 1998, 10, 546-552.

22. Cooper, J.M.; Morris, D.G.; Ryder, K.S. J. Chem. Soc., Chem. Comm. 1995, 697-700.

23. Saby, C.; Luong, J.H.T. Electroanalysis 1998, 10, 1193-1199.

24. Hiller, M.; Kranz, C.; Huber, J.; Bäuerle, P.; Schuhmann, W. Adv. Mater. 1996, 8, 219-222.

25. Baüerle, P.; Hiller, M.; Scheib, S.; Sokolowski, M.; Umbach, E. Adv. Mater. 1996, 8, 214-218.

26. Korri-Youssoufi, H.; Garnier, F.; Srivastava, P.; Godillot, P.; Yassar, A. J. Am. Chem. Soc. 1997, 119, 7388-7389.

27. Andrieux, C.P.; Audebert, P.; Hapiot, P.; Savéant, J.M. J. Am. Chem. Soc. 1990, 112, 2439-2440.

28. Garnier, F.; Korri Youssoufi, H.; Srivastava, P.; Yassar, A. J. Am. Chem. Soc. 1994, 116, 8813-8814

29. Heiduschka, P.; Göpel, W.; Beck, W.; Kraas, W.; Kienle, S.; Jung, G. Chem. Eur. J. 1996, 2, 667-672.

30. Wilchek, M.; Bayer, E.A. Anal. Biochem. 1988, 171, 1-32.

31. Pantano, P.; Kuhr, W.G. Anal. Chem. 1993, 65, 623-630.
32. Hoshi, T.; Anzai, J.; Osa, T. Anal. Chem. 1995, 67, 770-774.

33. Yang, S.T.; Witkowski, A.; Hutchins, R.S.; Scott, D.L.; Bachas, L.G. Electroanalysis 1998, 10, 58-60.

34. Leipert, D.; Heiduschka, P.; Mack, J.; Egelhaaf, H.J.; Oelkrug, D.; Jung, G. Angew. Chem. Int. Ed. 1998, 37, 2338-2340.

35. Cosnier, S.; Galland, B.; Gondran, C.; Le Pellec, A. Electroanalysis 1998,10, 808-813.

36. Torres-Rodriguez, L. M.; Roget, A.; Billon, M.; Livache, T.; Bidan, G. J. Chem. Soc., Chem. Commun. 1998, 1993-1994.

37. Cosnier, S.; Le Pellec, A. Electrochim. Acta 1999, 44, 18331836.

38. Cosnier, S.; Stoytcheva, M.; Senillou, A.; Perrot, H.; Leone, F.A. Anal. Chem. In press.

39. Frey, B.L.; Jordan, C. E.; Kornguth, S.; Corn, R. M. Anal. Chem. 1995, 67, 4452-4457.

40. Anzai , J.; Takeshita, H.; Hoshi, T; Osa, T. Denki Kagaku, 1995, 63, 1141-1142.

41. Anzai, J.; Nishimura, M. J. Chem. Soc. Perkin Trans. 2 1997, 1887-1889. 